\title{
High-dose proton-based radiation therapy in the management of spine chordomas: outcomes and clinicopathological prognostic factors
}

\author{
Ronny L. Rotondo, MD, ${ }^{1}$ Wendy Folkert, BA, ${ }^{1}$ Norbert J. Liebsch, MD, PhD, ${ }^{1}$ \\ Yen-Lin E. Chen, MD, ${ }^{1}$ Frank X. Pedlow, MD, ${ }^{2}$ Joseph H. Schwab, MD, MSc, ${ }^{2}$ \\ Andrew E. Rosenberg, MD, ${ }^{3}$ G. Petur Nielsen, MD, ${ }^{3}$ Jackie Szymonifka, MA, ${ }^{4}$ AI E. Ferreira, RN, ${ }^{2}$ \\ ${ }^{*}$ Francis J. Hornicek, MD, PhD, ${ }^{2}$ and Thomas F. DeLaney, MD'
}

Departments of ${ }^{1}$ Radiation Oncology, ${ }^{2}$ Orthopedic Surgery, and ${ }^{3}$ Pathology, and ${ }^{4}$ Division of Biostatistics, Massachusetts General Hospital, Harvard Medical School, Boston, Massachusetts

\begin{abstract}
OBJECT Spinal chordomas can have high local recurrence rates after surgery with or without conventional dose radiation therapy (RT). Treatment outcomes and prognostic factors after high-dose proton-based RT with or without surgery were assessed.

METHODS The authors conducted a retrospective review of 126 treated patients (127 lesions) categorized according to disease status (primary vs recurrent), resection (en bloc vs intralesional), margin status, and RT timing.

RESULTS Seventy-one sacrococcygeal, 40 lumbar, and 16 thoracic chordomas were analyzed. Mean RT dose was 72.4 GyRBE (relative biological effectiveness). With median follow-up of 41 months, the 5 -year overall survival (OS), local control (LC), locoregional control (LRC), and distant control (DC) for the entire cohort were $81 \%, 62 \%, 60 \%$, and $77 \%$, respectively. LC for primary chordoma was $68 \%$ versus $49 \%$ for recurrent lesions $(p=0.058)$. LC if treated with a component of preoperative RT was $72 \%$ versus $54 \%$ without this treatment $(p=0.113)$. Among primary tumors, $L C$ and LRC were higher with preoperative RT, $85 \%(p=0.019)$ and $79 \%(0.034)$, respectively, versus $56 \%$ and $56 \%$ if no preoperative RT was provided. Overall LC was significantly improved with en bloc versus intralesional resection $(72 \%$ vs $55 \%$, $p=0.016)$, as was $L R C(70 \%$ vs $53 \%, p=0.035)$. A trend was noted toward improved $L C$ and $L R C$ for R0/R1 margins and the absence of intralesional procedures.
\end{abstract}

CONCLUSIONS High-dose proton-based RT in the management of spinal chordomas can be effective treatment. In patients undergoing surgery, those with primary chordomas undergoing preoperative RT, en bloc resection, and postoperative RT boost have the highest rate of local tumor control; among 28 patients with primary chordomas who underwent preoperative RT and en bloc resection, no local recurrences were seen. Intralesional and incomplete resections are associated with higher local failure rates and are to be avoided.

http://thejns.org/doi/abs/10.3171/2015.3.SPINE14716

KEY WORDS chordoma; spine; sacrum; radiation therapy; surgery; oncology

$\mathrm{C}$ HORDOMAS arise in notochordal remnants in the midline from the skull base to sacrum. They are rare, with approximately 0.1 case $/ 100,000$ per year, accounting for approximately $4 \%$ of primary malignant bone tumors. Tumors are most commonly sacrococcygeal, followed by skull base and mobile spine locations. ${ }^{20}$ Surgery has been the standard local treatment, but margin- negative resection is not always feasible. Local recurrence rates are as high as 50\%-100\% with subtotal resection (STR) versus 0\%-53\% with en bloc, margin-negative resection. ${ }^{2,3,5,11-13,16,18,19,22,23}$

Strategies combining high-dose adjuvant RT and maximal resection have resulted in high local control (LC) rates. ${ }^{9,17} \mathrm{RT}$ using protons and/or photons is often neces-

ABBREVIATIONS CTV = clinical target volume; DC = distant control; EFS = event-free survival; GTR = gross-total resection; $L C=$ local control; $L R C=$ locoregional control; $\mathrm{NCI}$ CTCAE = National Cancer Institute Common Terminology Criteria for Adverse Events; OS = overall survival; RBE = relative biological effectiveness; RC = regional control; RT = radiation therapy; STR = subtotal resection.

SUBMITTED July 22, 2014. ACCEPTED March 25, 2015.

INCLUDE WHEN CITING Published online September 4, 2015; DOI: 10.3171/2015.3.SPINE14716.

* Drs. Hornicek and DeLaney share senior authorship of this work. 
sary, because a negative margin resection is often impossible due to adjacent critical structures. Medical (drug) treatments for chordomas have limited efficacy. ${ }^{21}$ Reports of durable tumor control without surgery using high-dose radiation with protons ${ }^{7}$ or carbon ions are encouraging. ${ }^{14}$

Because various surgical approaches have been used, ranging from intralesional to en bloc, the authors analyzed their influence on LC, particularly in the setting of highdose adjuvant RT. Massachusetts General Hospital staff prefer to administer some preoperative RT to reduce iatrogenic tumor seeding associated with surgical violation of the tumor. As a referral center for proton radiation, the staff also treat patients referred after surgery at other institutions with only postoperative RT, allowing an assessment of RT timing on outcome.

\section{Methods}

After receiving Partners Healthcare institutional review board approval, the authors conducted a retrospective review of patients with thoracic, lumbar, and sacrococcygeal chordomas treated with high-dose proton-based RT with or without surgery. By using departmental databases, 158 patients were identified who were treated between 1982 and 2011, of whom 126 (127 lesions) were included in this study. Thirty-two patients were excluded for the following reasons: 21 patients with primary disease treated nonsurgically with definitive proton-based radiotherapy (subjects of a separate study $\left.{ }^{7}\right), 7$ patients treated with photon radiation, 3 patients presenting with metastases, and 1 patient treated at another proton facility.

Hospital records were reviewed to assess patient, treatment, and toxicity characteristics; local control (LC), regional control (RC), locoregional control (LRC), and distant control (DC) of the tumor; and overall survival (OS). LC was considered the absence of tumor at the primary site, $\mathrm{RC}$ was the absence of tumor in the adjacent regional tissues (i.e., adjacent vertebra or draining lymph nodes), LRC was the absence of any local or regional tumor, and DC was the absence of any distant metastatic disease. Patients followed at the study center were evaluated every 6 months after treatment for 4 years and then yearly thereafter with cross-sectional imaging of the primary site, and chest imaging (chest radiography alternating with CT) for the first 4 years, then yearly chest radiography thereafter. These follow-up recommendations were communicated to referring physicians evaluating patients who were not able to return to the study center for follow-up. For patients who could not return for or were lost to follow-up, reliance was placed on correspondence from physicians, imaging reports, patient telephone interviews, or all of these. During the interviews, patients were asked if their tumor had recurred locally or distantly, as well as whether they had ongoing pain, required medication for pain, and whether they had neurological deficits that had progressed since the time of the last follow-up evaluation. Patients were also asked to forward relevant medical records to the study center office. Patients were categorized by disease status at referral (primary vs recurrent after surgery), surgery (en bloc vs intralesional), gross-total resection (GTR) versus STR, margin status (R0, R1, R2), and RT (pre- and postoperative vs exclusively postoperative RT), or RT without additional surgery for recurrent chordomas. We excluded patients who had undergone prior radiotherapy. Table 1 lists patient and tumor characteristics.

\section{Surgery Definitions}

En bloc resection was defined as tumor removal in a single specimen without tumor violation; intralesional resection included piecemeal resection or unplanned tumor violation. R0 margins were defined as the absence of tumor on the pathologically assessed surgical margins of an en bloc resection specimen. R1 margins had microscopic tumor at the surgical margins of a grossly complete en bloc resection or a visibly complete intralesional resection without any evident residual tumor on postoperative imaging. R2 resections were incomplete tumor resections. Because this series spans many years and surgery was performed at various centers, the surgical procedures and margins reflect the anatomical level of disease; the choice of the surgeon, patient, or both; as well as the philosophy of the treating center. Patients presenting with recurrent disease and receiving definitive RT without additional surgery had either declined surgery or were not amenable to resection with acceptable morbidity.

\section{Radiation Therapy Approaches}

RT was delivered in most patients with a combination

TABLE 1. Patient and tumor characteristics*

\begin{tabular}{|c|c|}
\hline Characteristic & Value \\
\hline No. of patients & 126 \\
\hline No. of chordomas & $127 \dagger$ \\
\hline \multicolumn{2}{|l|}{ Sex } \\
\hline Men & $79(62.2)$ \\
\hline Women & $48(37.8)$ \\
\hline \multicolumn{2}{|l|}{ Age at diagnosis (yrs) } \\
\hline Mean (range) & $53.2(5-88)$ \\
\hline \multicolumn{2}{|l|}{ Histology } \\
\hline Chondroid chordoma & $28(22.05)$ \\
\hline Nonchondroid chordoma & 99 (77.95) \\
\hline \multicolumn{2}{|l|}{ Site of origin } \\
\hline Thoracic spine & $16(12.6)$ \\
\hline Lumbar spine & $40(31.5)$ \\
\hline Sacrococcygeal & $71(55.9)$ \\
\hline \multicolumn{2}{|l|}{ Presentation } \\
\hline Primary & $95(74.8)$ \\
\hline Recurrent after prior op & $32(25.2)$ \\
\hline \multicolumn{2}{|l|}{ Spinal canal extension } \\
\hline Extraspinal & $32(25.2)$ \\
\hline Intraspinal & $88(69.3)$ \\
\hline Unknown & $7(5.5)^{\prime}$ \\
\hline \multicolumn{2}{|l|}{ No. of vertebral levels involved } \\
\hline Single & $50(39.37)$ \\
\hline Multiple & 77 (60.63) \\
\hline \multicolumn{2}{|l|}{ Maximum tumor size $(\mathrm{cm})$} \\
\hline Mean (range) & $7.0(1.6-21.7)$ \\
\hline
\end{tabular}

* Values expressed as number of lesions (\%) unless noted otherwise.

† One patient had synchronous lumbar and sacrococcygeal chordomas. 
of photons and 3D conformal passively scattered protons. RT dose and fractionation were according to protocol or, in the absence of active protocols for which the patient was eligible at the time of treatment, according to treating physician prescription. Doses are expressed as relative biological effectiveness (RBE)-weighted absorbed dose, GyRBE, which equals 1 proton Gy $\times$ the estimated proton $\mathrm{RBE}$ of 1.1. Patients were generally treated with daily external beam fractions of 1.8-2 GyRBE.

For patients receiving preoperative RT, 19.8-50.4 GyRBE was given, depending on the perceived risk of perioperative wound complications. Mobile spine tumors were generally treated with 50.4 Gy preoperative RT, with surgery 1 month later, unless the patient had impending spinal cord compression, in which case 19.8-20 Gy was given, followed by immediate surgery. Because sacrococcygeal tumors present more challenging wound healing problems (even after surgery alone), sacral lesions received only 19.8-20 Gy, followed by surgery within 2 weeks. Patients receiving preoperative RT were also planned to receive additional postoperative boost RT to bring the tumor bed dose to approximately 70 GyRBE, the high dose required for chordomas. The entire RT dose was not delivered preoperatively, because of the prohibitive risk of wound healing problems with such high radiation doses. The preoperative RT clinical target volume (CTV1) typically encompassed gross disease, the involved vertebral levels (and any disease within the spinal canal), and 1 level above and below, with a radial margin on any extraosseous extension of $1-1.5 \mathrm{~cm}$ or up to a constraining fascial or anatomical barrier. In the sacrococcygeal region, the CTV1 could also extend laterally to include possible routes of subclinical extension, such as piriformis and sacrococcygeus musculature and sacro-spinous ligaments.

After preoperative RT, patients underwent maximal resection. As previously reported, for tumors of the thoracolumbar region abutting the dura, electron-based intraoperative RT or dural plaque brachytherapy was used to boost involved dura. ${ }^{8}$

\section{Postoperative RT Therapy}

After wound healing, patients underwent postoperative boost radiation planning $\mathrm{CT}$, with lumbar myelographic contrast instillation for tumors above L-3 (i.e., in proximity to the spinal cord). Postoperative boost RT was delivered using the shrinking field technique to maximize the final radiation dose to the areas of highest residual tumor burden. The initial CTV1 received approximately 50.4 GyRBE, either completely as preoperative radiation or as combined preoperative and initial postoperative phase treatment in patients who had only received 19.8 GyRBE preoperatively. In patients receiving preoperative RT, no attempt was made to include all surgically manipulated tissues and drain sites in their postoperative treatment volumes. After the CTV1 had received approximately 50.4 Gy, the radiation target volume was reduced to encompass the tumor bed and a 5-mm CTV margin, treating this boost volume to approximately 70.2 GyRBE. A third postoperative phase was then used in cases of STR to treat gross residual disease to a total dose of approximately 77.4 GyRBE.
In patients treated with only postoperative RT, the initial CTV1 treated to approximately 50.4 GyRBE included the tissues described above in the preoperative CTV1, but also included surgically manipulated tissues, including scars, drain sites, and stabilization hardware that could be encompassed in a treatable radiation volume. Phase 2 of postoperative RT included the tumor bed plus a margin of $5 \mathrm{~mm}$ to a dose of approximately $70.2 \mathrm{~Gy}$. When applicable, such as in cases of STR, a third phase was used to treat any gross residual disease to a total dose of approximately 77.4 GyRBE.

LC following GTR was defined as the absence of disease on follow-up imaging. After STR or biopsy only, the regression or stabilization of residual disease on followup imaging was considered LC. RC was defined as the absence of nodal metastases or surgical tract recurrence. Toxicity was recorded and graded according to National Cancer Institute Common Terminology Criteria for Adverse Events (NCI CTCAE) version 4.0 with attention to neuropathy/myelopathy, bone necrosis or insufficiency fractures, wound complications, and fibrosis.

\section{Statistical Methods}

Follow-up and other events were measured from the end of the last treatment (surgery or RT). OS included death from any cause. Event-free survival (EFS) measured any event, including local failure, regional failure, distant failure, or death. For LC, RC, and DC, only failures of the respective type were counted as events. Univariate Cox regression models were performed for OS, LC, RC, LRC, and DC. Clinical outcomes were evaluated using the Kaplan-Meier method, and all subgroups were compared by stratified log-rank test. All $p$ values were 2 -tailed; $p$ $<0.05$ was considered statistically significant. Statistical analysis was performed using SAS Software (version 9.2; SAS Institute). A multivariate analysis was performed to determine which factors serve as confounders and whether some of these associations might become insignificant when all of these variables were considered together.

\section{Results}

\section{Treatment Characteristics}

Patient treatment is outlined in Table 2. Although approximately half of the definitive resections were en bloc, 79 lesions (62.20\%) had undergone an intralesional procedure at some point in treatment; 45 tumors $(35.43 \%)$ had undergone no intralesional procedures.

Intraoperative ${ }^{90} \mathrm{Y}^{32} \mathrm{P}$ dural plaque brachytherapy was used in 7 patients (median dose $10 \mathrm{~Gy}$, range 7.5-11 Gy). Electron intraoperative RT was delivered in 4 patients (median dose $10 \mathrm{~Gy}$, range 3.6-12 Gy) while 4 patients received ${ }^{192} \mathrm{I}$ low-dose-rate interstitial brachytherapy (median 42 Gy, range $27-50$ Gy).

\section{Treatment Outcomes: Entire Cohort and by Histological Characteristics}

Median follow-up duration after RT was 47 months, with median time since last follow-up of 5.7 months. Of the 98 patients alive as of last contact, $83(85 \%)$ had $\geq 1$ year of follow-up, 64 (65\%) had $\geq 2$ years, and $56(57 \%)$ 
TABLE 2. Treatment characteristics

\begin{tabular}{|c|c|}
\hline Characteristic & Value* \\
\hline \multicolumn{2}{|l|}{ Op (\%) } \\
\hline En bloc & $62(48.82)$ \\
\hline Intralesional & $62(48.82)$ \\
\hline Unknown & $3(2.36)$ \\
\hline \multicolumn{2}{|l|}{ Extent of resection (\%) } \\
\hline GTR & $97(76.38)$ \\
\hline STR & $30(23.62)$ \\
\hline \multicolumn{2}{|l|}{ Margin status (\%) } \\
\hline RO & $34(26.77)$ \\
\hline $\mathrm{R} 1$ & $57(44.88)$ \\
\hline $\mathrm{R} 2$ & $30(23.62)$ \\
\hline Unknown & $6(4.72)$ \\
\hline \multicolumn{2}{|l|}{ No. of ops prior to definitive resection (\%) } \\
\hline 0 & $66(51.97)$ \\
\hline 1 & 37 (29.13) \\
\hline 2 & $17(13.39)$ \\
\hline$\geq 3$ & $7(5.51)$ \\
\hline \multicolumn{2}{|l|}{ Intralesional resection at any time (\%) } \\
\hline Yes & $79(62.20)$ \\
\hline No & $45(35.43)$ \\
\hline Unknown & $3(2.36)$ \\
\hline \multicolumn{2}{|l|}{ Treatment category } \\
\hline \multicolumn{2}{|l|}{ Primary tumors (\%) } \\
\hline Op \& postop RT & $51(40.16)$ \\
\hline Op \& pre-/postop RT & $44(34.65)$ \\
\hline \multicolumn{2}{|l|}{ Recurrent tumors (\%) } \\
\hline Op \& postop RT & $7(5.51)$ \\
\hline Op \& pre-/postop RT & $16(12.60)$ \\
\hline Definitive RT for recurrent tumor & $9(7.09)$ \\
\hline \multicolumn{2}{|l|}{ Radiation dose characteristics (GyRBE) } \\
\hline \multicolumn{2}{|l|}{ Entire cohort (range) } \\
\hline Total dose & $72.4(46.3-83.6)$ \\
\hline Photon dose & $32.5(0-58.0)$ \\
\hline Proton dose & $39.9(18.0-77.4)$ \\
\hline \multicolumn{2}{|l|}{ Postop RT cohort (range) } \\
\hline Total dose & $73.7(63.0-82.1)$ \\
\hline \multicolumn{2}{|l|}{ Pre-/postop RT cohort (range) } \\
\hline Total dose & $71.0(46.3-83.6)$ \\
\hline Preop dose & $30.3(8.0-50.4)$ \\
\hline Postop dose & $40.8(18.0-70.2)$ \\
\hline \multicolumn{2}{|c|}{ Definitive RT for recurrence cohort (range) } \\
\hline Total dose & $76.0(70.0-82.1)$ \\
\hline
\end{tabular}

$\mathrm{R} 0$ = negative margin, $\mathrm{R} 1$ = microscopically positive margin, $\mathrm{R} 2$ = macroscopically positive margin.

* Data are presented as number of lesions (\%) or mean (range) absorbed dose expressed as GyRBE.

had $\geq 3$ years of follow-up. The latest follow-up date was truncated at February 28, 2011. Of the 98 patients alive, 35 were last known to be alive prior to January 1, 2010, and 29 of those 35 were last seen prior to January 1, 2009. Treatment outcomes are shown in Fig. 1 and Table 3; 5-year OS was $81 \%$ (95\% CI 69\%-88\%), LC 62\% (95\% CI $50 \%-72 \%)$, RC 92\% (95\% CI 83\%-96\%), LRC $60 \%$ (95\% CI 48\%-70\%), and DC $77 \%$ (95\% CI 66\%-84\%). Table 4 summarizes patient status and events. At the time of analysis, 111 patients were alive with 69 patients
$(62.16 \%)$ without evidence of disease, 8 (7.20\%) progression-free, and 34 (30.63\%) living with disease. Table 4 also lists recurrences. Of the 38 patients with local failure, 16 $(42.11 \%)$ were isolated, while $6(15.79 \%)$ were combined with regional failure, $15(39.47 \%)$ had distant failure, and $1(2.63 \%)$ both locoregional and distant failure. As listed in Table 3, no significant differences occurred in OS, LC, LRC, RC, or DC (data not shown) among chordoma histological subtypes, whether chondroid and nonchondroid. Of note, there was no significant difference according to anatomical location, spinal canal extension, number of vertebral levels involved, total radiation dose (although the range of total doses was quite narrow), or maximum tumor size (data not shown).

\section{Outcomes: Primary Versus Recurrent Presentation}

For patients with primary disease, 5-year OS was $82 \%$ (95\% CI 68\%-91\%) versus 78\% (95\% CI 54\%-90\%) for recurrent disease $(\mathrm{p}=0.342)$. There was a strong trend for improved LC and LRC with primary disease (Table 3). Similarly, the DC rate with primary disease was $80 \%$ (95\% CI 68\%-88\%) versus 67\% (95\% CI 44\%-83\%) for recurrent disease $(\mathrm{p}=0.106)$. There was no significant difference in RC between primary and recurrent presentation $(p=0.691)$. Figure 2 illustrates LC for primary and recurrent disease.

\section{Outcomes: Surgery Type, Extent, and Margin Status}

In patients undergoing en bloc resection, 5-year OS and LC rates were $86 \%(95 \%$ CI 67\%-95\%) and $72 \%(95 \%$ CI 52\%-85\%), respectively. Patients who underwent intralesional surgeries had 5-year OS and LC rates of $78 \%$ (95\% CI 62\%-88\%) and 55\% (95\% CI 39\%-68\%), respectively. There was a trend toward improved OS and a statistically significant improvement in LC (Fig. 3) and LRC with en bloc versus an intralesional procedure, with $\mathrm{p}$ values of $0.081,0.016$, and 0.035 , respectively. No significant difference occurred in RC, LRC, or DC between the 2 groups. Patients who had no intralesional surgical procedure trended toward higher 5-year OS, LC, and LRC rates (Table 3 ) than those who had any intralesional procedure. Moreover, when the effect of the number of prior surgical interventions before definitive surgical management on outcomes was examined, LC, RC, and LRC were found to be significantly worse with an increasing number of prior surgical interventions, with hazard ratios (HRs) of 1.44 (95\% CI 1.03\%-2.02\%; p = 0.034), $1.99(95 \% \mathrm{CI}$ $1.01 \%-3.90 \% ; \mathrm{p}=0.046)$, and $1.54(95 \%$ CI $1.11 \%-2.12 \%$; $\mathrm{p}=0.009)$, respectively.

In patients who underwent GTR, the 5-year OS rate was $86 \%$ (95\% CI 72\%-93\%) versus 68\% (95\% CI 43\%$84 \%)$ for STR $(\mathrm{p}=0.190)$. LC rates were $64 \%(95 \% \mathrm{CI}$ $50 \%-75 \%)$ for GTR versus $55 \%$ (95\% CI 32\%-73\%) for STR ( $p=0.289)$, respectively. There were no significant differences between GTR versus STR for the RC, LRC, and DC end points.

For patients with R0, R1, and R2 margin status, 5-year OS rates were $76 \%(95 \%$ CI $40 \%-92 \%), 91 \%$ (95\% CI 77\%-97\%), and 66\% (95\% CI 40\%-83\%). The 5-year LC rates were $72 \%$ (95\% CI 40\%-89\%), 64\% (95\% CI 47\%- 
A
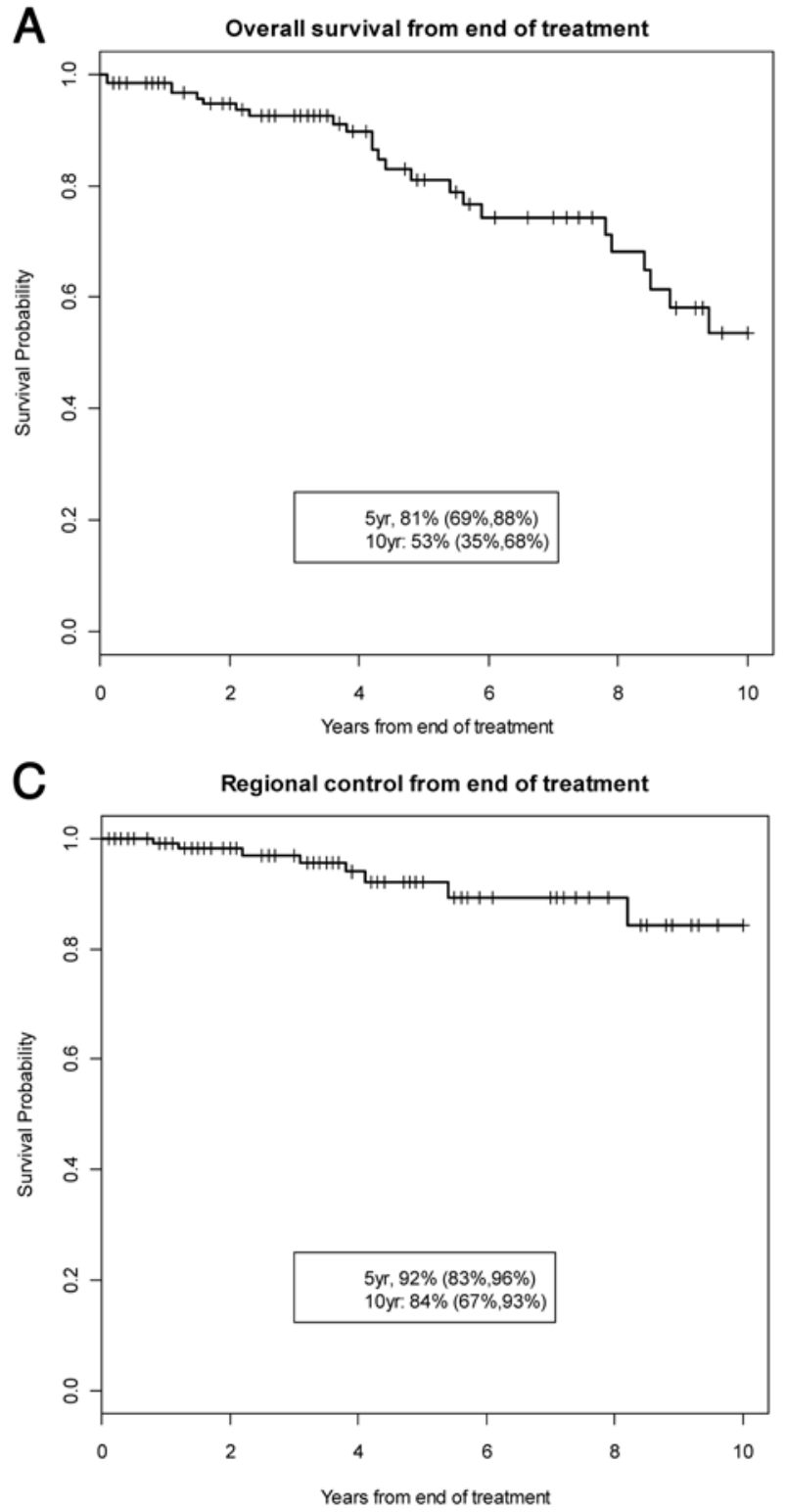

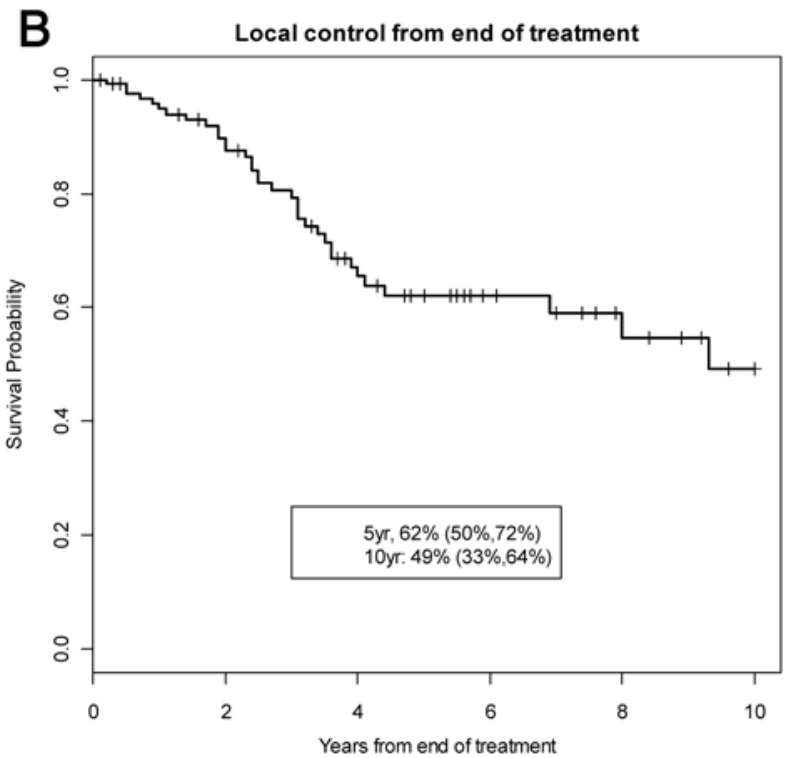

D

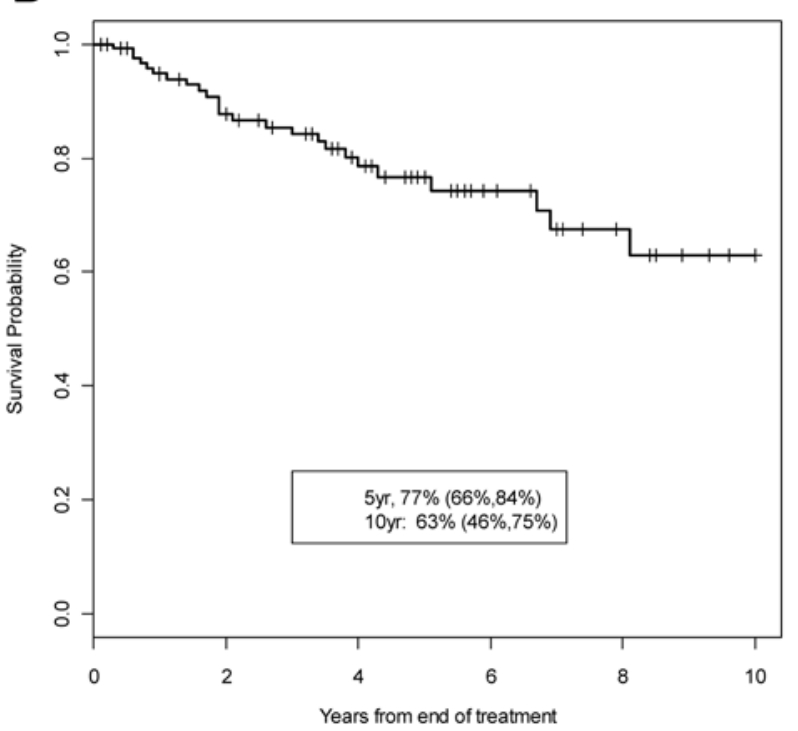

FIG. 1. Kaplan-Meier estimate of outcomes for entire cohort, including OS (A), LC (B), RC (C), and DC (D). Five- and 10-year outcome percentages are shown, along with $95 \% \mathrm{Cls}$ (in parentheses).

$77 \%$ ), and $53 \%$ (95\% CI 30\%-71\%) for R0, R1, and R2 margin status, respectively. When comparing R0/R1 versus R2 margin status, a significant improvement occurred in OS (5-year OS $88 \%$ vs $66 \%, p=0.017)$ and a strong trend toward improved LC (5-year LC $66 \%$ vs 53\%, p = 0.074). There were no significant differences in RC, LRC, or DC by margin status (Table 3 ).

\section{Outcomes: Preoperative/Postoperative RT, Only Postoperative RT, and Definitive RT (Recurrent Disease)}

For primary tumor patients who underwent only postoperative RT, the 5-year OS, LC, RC, LRC, and DC rates were $80 \%$ (95\% CI $57 \%-91 \%$ ), $56 \%$ (95\% CI 37\%-71\%), $97 \%$ (95\% CI $78 \%-100 \%), 55 \%$ (95\% CI $36 \%-71 \%$ ), and $82 \%$ (95\% CI 65\%-91\%), respectively. Patients with primary tumors who underwent combined pre- and postop- erative RT had 5-year OS, LC, RC, LRC, and DC rates of $85 \%$ (95\% CI 63\%-95\%), 85\% (95\% CI 59\%-95\%), 88\% (95\% CI 64\%-96\%), $79 \%$ (95\% CI 55\%-91\%), and $78 \%$ (95\% CI 56\%-90\%), respectively. For patients with recurrent tumors who underwent only postoperative RT, the 5-year OS, LC, RC, LRC, and DC rates were 83\% (95\% CI $27 \%-97 \%), 44 \%$ (95\% CI 7\%-78\%), $100 \%$ (95\% CI $100 \%-100 \%), 44 \%$ (95\% CI $7 \%-78 \%$ ), and $57 \%$ (95\% CI $8 \%-89 \%$ ), respectively. Patients with recurrent tumors who underwent combined pre- and postoperative RT had 5-year OS, LC, RC, LRC, and DC rates of 71\% (95\% CI 33\%90\%), $47 \%$ (95\% CI 17\%-73\%), $77 \%$ (95\% CI 31\%-94\%), $47 \%$ (95\% CI 17\%-73\%), and $92 \%$ (95\% CI 57\%-99\%), respectively. For patients with recurrent tumors who received definitive RT, the 5-year OS, LC, RC, LRC, and DC rates were $83 \%$ (95\% CI 27\%-97\%), 56\% (95\% CI 20\%-80\%), 
TABLE 3. Outcomes for all patients

\begin{tabular}{|c|c|c|c|c|c|c|}
\hline \multirow[b]{2}{*}{ Patient Characteristic } & \multicolumn{2}{|c|}{ OS (126 patients) } & \multicolumn{2}{|c|}{ LC (127 lesions) } & \multicolumn{2}{|c|}{ LRC (127 lesions) } \\
\hline & $5-\mathrm{Yr} \%(95 \% \mathrm{Cl})$ & p Value & $5-\mathrm{Yr} \%(95 \% \mathrm{Cl})$ & p Value & $5-\mathrm{Yr} \%(95 \% \mathrm{Cl})$ & $\mathrm{p}$ Value \\
\hline Entire cohort $(n=127)^{*}$ & $81(69-88)$ & & $62(50-72)$ & & $60(48-70)$ & \\
\hline Histological subtype & & 0.268 & & 0.487 & & 0.436 \\
\hline Chondroid chordoma $(n=28)$ & $100(100-100)$ & & $52(26-73)$ & & $52(26-73)$ & \\
\hline Nonchondroid chordoma $(n=99)$ & $76(63-85)$ & & $64(50-75)$ & & $62(48-73)$ & \\
\hline Presentation & & 0.342 & & 0.058 & & 0.120 \\
\hline Primary $(n=95)$ & $82(68-91)$ & & $68(53-78)$ & & $65(50-76)$ & \\
\hline Recurrent $(n=32)$ & $78(54-90)$ & & $49(28-67)$ & & $49(28-67)$ & \\
\hline Surgery & & 0.081 & & $0.016 \ddagger$ & & 0.035 \\
\hline En bloc $(n=62)$ & $86(67-95)$ & & $72(52-85)$ & & $70(37-88)$ & \\
\hline Intralesional $(n=62)$ & $78(62-88)$ & & $55(39-68)$ & & $55(35-72)$ & \\
\hline Extent of resection & & 0.190 & & 0.289 & & 0.347 \\
\hline GTR $(n=97)$ & $86(72-93)$ & & $64(50-75)$ & & $62(48-73)$ & \\
\hline $\operatorname{STR}(n=30)$ & $68(43-84)$ & & $55(32-73)$ & & $54(31-73)$ & \\
\hline Intralesional procedure† & & 0.147 & & 0.086 & & 0.092 \\
\hline No $(n=45)$ & $89(61-97)$ & & $68(43-84)$ & & $68(43-84)$ & \\
\hline Yes $(n=79)$ & $79(65-88)$ & & $58(44-70)$ & & $55(41-67)$ & \\
\hline Margin status & & $0.017 \ddagger \S$ & & $0.074 \S$ & & $0.350 \S$ \\
\hline $\mathrm{RO}(\mathrm{n}=34)$ & $76(40-92)$ & & $72(40-89)$ & & $69(39-86)$ & \\
\hline $\mathrm{R} 1(\mathrm{n}=57)$ & 91 (77-97) & & $64(47-77)$ & & $60(43-74)$ & \\
\hline$R 2(n=30)$ & $66(40-83)$ & & $53(30-71)$ & & $54(31-73)$ & \\
\hline
\end{tabular}

$100 \%$ (95\% CI 100\%-100\%), 56\% (95\% CI 20\%-80\%), and $40 \%$ (95\% CI 10\%-70\%), respectively. When comparing outcomes examined in this study between the 5 RT treatment categories listed above, patients with primary disease who underwent surgery with pre- and postoperative RT showed a trend toward the highest LC $(p=0.074)$. When further examining the cohort of patients presenting

\section{TABLE 4. Patient status and events}

\begin{tabular}{cc}
\hline \multicolumn{1}{c}{ Variable } & No. \\
\hline Status & 15 \\
Deaths & 11 \\
Disease & 1 \\
Other cause & 2 \\
Other cancer & 1 \\
Unknown & 111 \\
Alive & 69 \\
No evidence of disease & 8 \\
Progression-free & 34 \\
w/ disease & \\
Failures & 38 \\
Local & 8 \\
Regional & 26 \\
Distant & \\
Distant failure sites & 9 \\
Bone & 9 \\
Lung & 3 \\
Bone \& lung & 3 \\
Liver &
\end{tabular}

with primary disease, 5-year LC and LRC rates were significantly improved when patients received a component of preoperative RT versus postoperative RT alone (Fig. 4). The 5-year LC and LRC rates for primary patients who received preoperative RT were $85 \%$ (95\% CI 59\%-95\%) and $79 \%$ (95\% CI 55\%-91\%), respectively, versus $56 \%$ (95\% CI

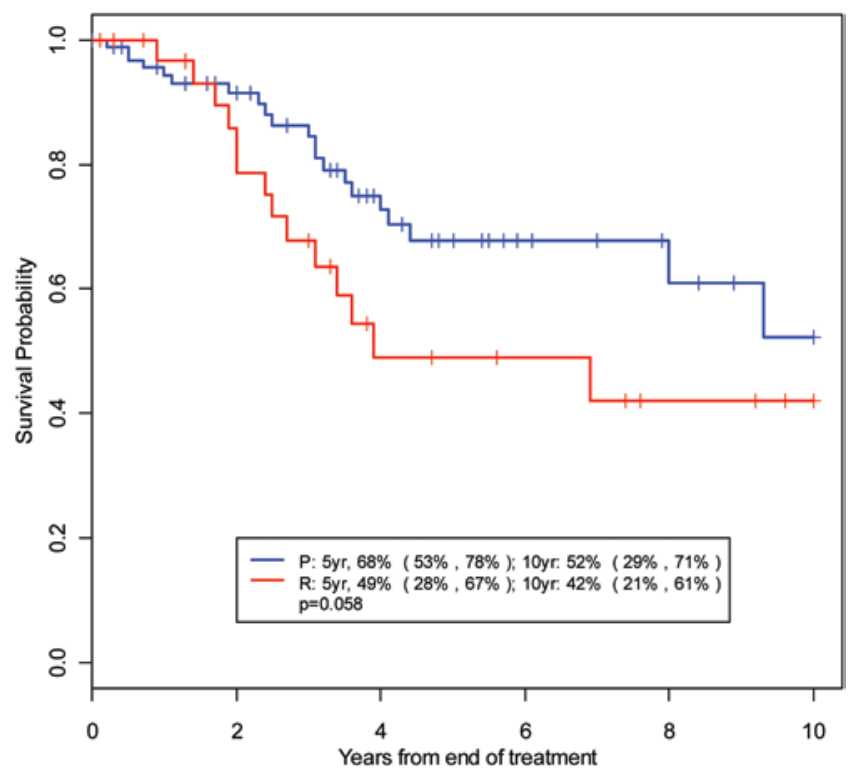

FIG. 2. Kaplan-Meier estimate of LC according to disease presentation. Five- and 10 -year LC percentages are shown along with $95 \% \mathrm{Cls}$ (in parentheses). $\mathrm{P}=$ primary disease; $\mathrm{R}=$ recurrent disease. 


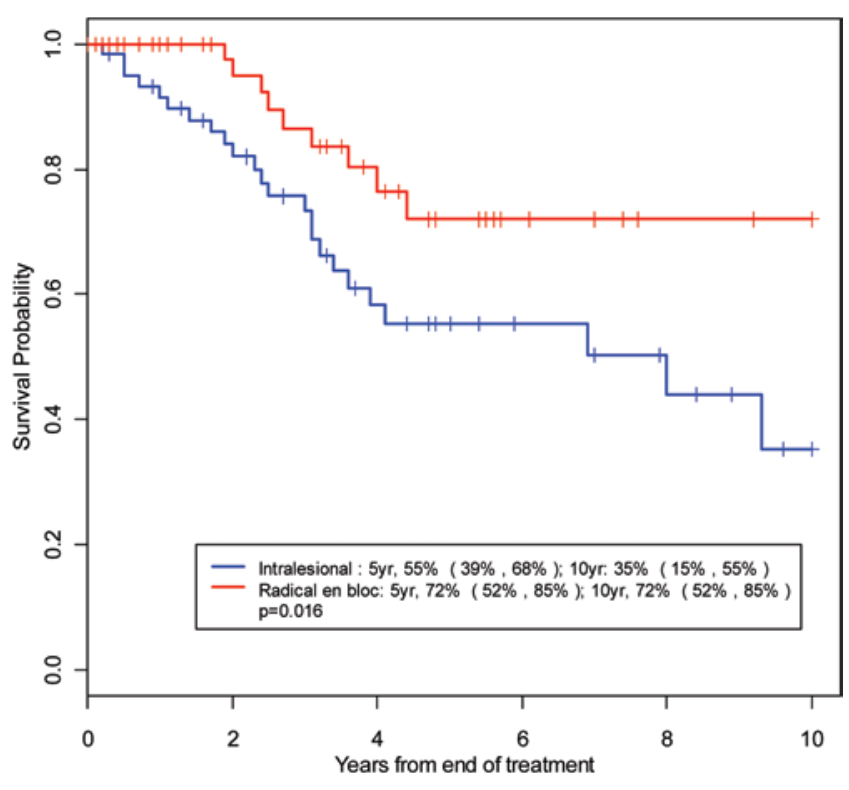

FIG. 3. Kaplan-Meier estimate of LC according to surgical extent. Five- and 10 -year LC percentages are shown along with $95 \% \mathrm{Cls}$ (in parentheses).

$37 \%-71 \%)$ and $56 \%(95 \%$ CI $37 \%-71 \%)$ for patients who received postoperative RT alone ( $\mathrm{p}=0.019$ and 0.034 , respectively). Notably, none of the 28 patients who were treated for primary tumors with preoperative radiation and who underwent an en bloc resection suffered a local recurrence.

\section{Multivariate Analysis}

When multivariate analysis (Table 5) was performed, there was a trend toward improved LC with preoperative RT $(p=0.11)$ and radical en bloc resection $(p=0.07)$, with a trend toward improved EFS with primary tumors $(\mathrm{p}=$

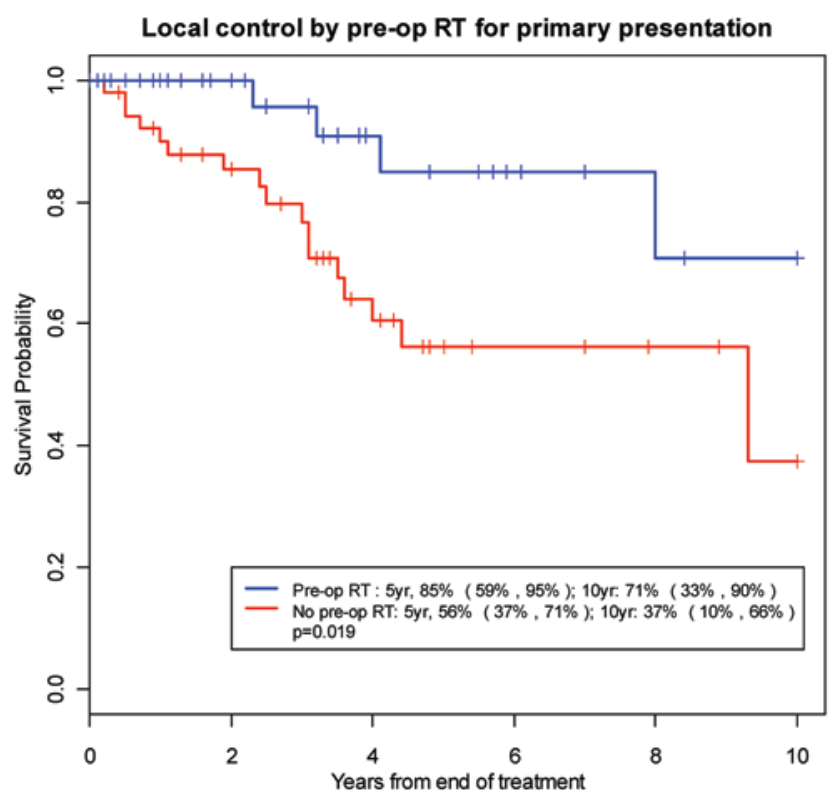

0.11). On stepwise selection modeling, radical en bloc resection was significantly associated with improved LC (p $=0.04)$ and LRC $(\mathrm{p}=0.04)$.

\section{Outcomes: Neurological Status}

Compared with neurological status at presentation, at last follow-up improvement was noted in 7 patients (5.6\%), stability in 61 (48.4\%), deterioration in $54(42.9 \%)$, and unknown status in 4 (3.2\%). Deterioration in neurological status was attributed to surgery in 23 patients $(18.25 \%)$, to radiotherapy in 9 (7.14\%), and to progressive local disease in $22(17.46 \%)$. No radiation-associated myelitis was observed.

\section{Outcomes: Treatment-Related Complications ( $\geq$ Grade 3)}

Patient records were reviewed to document treatmentrelated complications $\geq$ Grade 3 (NCI CTCAE, version 4.0), tabulated in Table 6. Because of the challenges of retrospective assessment of toxicity, particularly in a referral population, we would also direct readers to our prior publication of a prospective Phase 2 study of high-dose proton-based radiation in patients with spinal sarcomas in which the actuarial rate of radiation-associated Grade 3-4 toxicity at 8 years was $13 \% .^{10}$

\section{Discussion}

This analysis, albeit with the recognized shortcomings of retrospective studies, represents one of the larger reports of outcomes for mobile spinal and sacral chordomas. Because of the rarity of this disease and the desire to include as many patients as possible to have the largest possible data set for analysis, it is important to recognize that patients were treated over a 29 -year period, during which imaging, surgical, and radiation techniques evolved and improved. For example, the original proton facility in

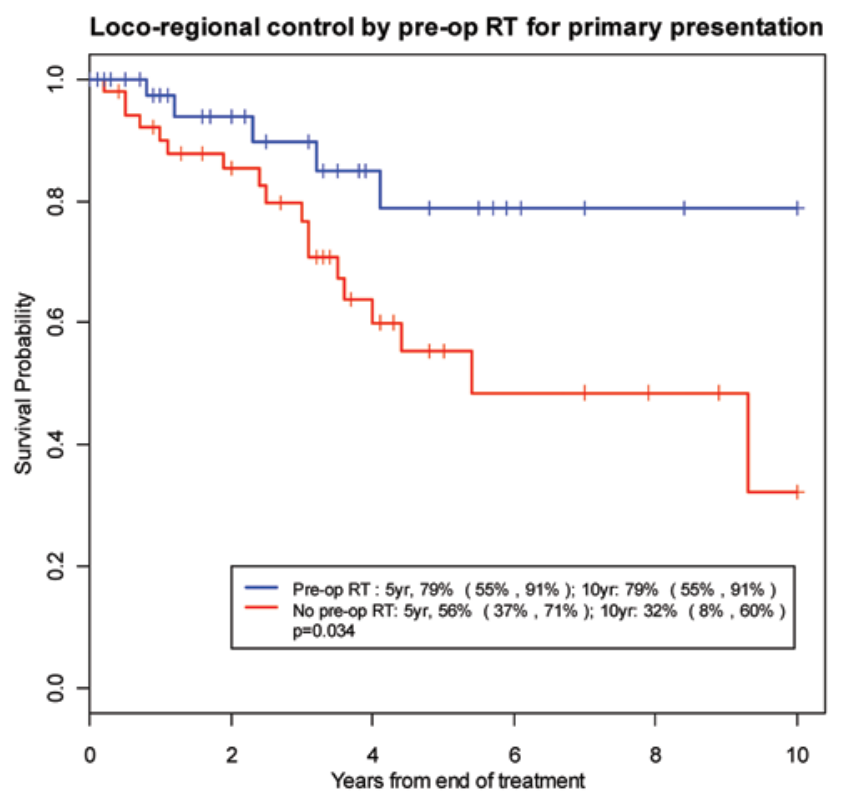

FIG. 4. Kaplan-Meier estimate of LC (left) and LRC (right) for patients with primary presentation who received a component of preoperative RT versus postoperative RT alone. Five- and 10-year LC percentages are shown along with $95 \%$ Cls (in parentheses). 
TABLE 5. Multivariate analysis of prognostic factors

\begin{tabular}{|c|c|c|c|c|c|c|c|c|}
\hline \multirow[b]{2}{*}{ Multivariate Variable } & \multicolumn{2}{|l|}{ OS } & \multicolumn{2}{|l|}{ LC } & \multicolumn{2}{|l|}{ LRC } & \multicolumn{2}{|l|}{ EFS } \\
\hline & $\mathrm{HR}(95 \% \mathrm{Cl})$ & p Value & $\mathrm{HR}(95 \% \mathrm{Cl})$ & $p$ Value & $\mathrm{HR}(95 \% \mathrm{Cl})$ & $p$ Value & $\mathrm{HR}(95 \% \mathrm{Cl})$ & $p$ Value \\
\hline Preop RT & $0.90(0.39-2.1)$ & 0.80 & $0.55(0.27-1.1)$ & 0.11 & $0.60(0.30-1.2)$ & 0.15 & $0.89(0.51-1.5)$ & 0.67 \\
\hline Primary lesion & $0.83(0.3-2.1)$ & 0.70 & $0.60(0.28-1.3)$ & 0.19 & $0.66(0.31-1.4)$ & 0.28 & $0.59(0.31-1.1)$ & 0.11 \\
\hline $\mathrm{R} 2$ resection & $1.7(0.76-3.9)$ & 0.19 & $1.2(0.59-2.6)$ & 0.56 & $1.2(0.56-2.4)$ & 0.70 & $1.6(0.85-2.8)$ & 0.15 \\
\hline Sacrococcygeal site & $1.1(0.42-2.8)$ & 0.86 & $1.0(0.46-2.3)$ & 0.95 & $1.0(0.47-2.2)$ & 0.97 & $0.95(0.50-1.8)$ & 0.87 \\
\hline Radical en bloc & $0.49(0.15-1.5)$ & 0.22 & $0.41(0.15-1.1)$ & 0.07 & $0.44(0.18-1.1)$ & 0.08 & $0.65(0.32-1.3)$ & 0.24 \\
\hline \multicolumn{9}{|l|}{ Stepwise selection modeling } \\
\hline Radical en bloc & NS & - & $0.40(0.16-0.96)$ & 0.04 & $0.43(0.19-0.98)$ & 0.04 & NS & - \\
\hline
\end{tabular}

NS = not significant.

which these patients were treated was in a physics laboratory with a horizontal, nonrotational beam of limited energy that could not treat patients above L-3, whereas starting in 2001, patients could be treated at a newer hospital-based facility with a rotational gantry with sufficient energy and field matching (patching) capability to treat any site in the spinal axis. In spite of high-dose photon/proton radiation and surgery (for 119 of the 126 patients), LC for the entire cohort was still only $62 \%(95 \%$ CI $50 \%-72 \%)$ at 5 years and $49 \%(95 \%$ CI 33\%-64\%) at 10 years; results in particular subsets, as noted below, however, were much better. Distant metastases, although less common, occurred in $23 \%$ of patients by 5 years.

Several important observations emerged from our analysis. There was a strong trend toward improved local tumor control in patients with primary tumors, i.e., $68 \%$ at 5 years compared with only $49 \%$ in patients presenting with locally recurrent tumor $(\mathrm{p}=0.058)$. This emphasizes the critical importance of concerted efforts to secure LC at initial presentation. Notably, en bloc resections were significantly more effective than intralesional resections with 5-year LC values of $72 \%$ and $55 \%$, respectively $(\mathrm{p}=$
0.016), consistent with the findings of a large systematic review. ${ }^{6}$ An increasing number of surgical procedures prior to definitive surgery was associated with worse LC with an HR of 1.44 ( $p=0.034)$, suggesting that intralesional surgical biopsy procedures, which can seed surgical tracts, be avoided $^{1}$ in favor of CT-directed core biopsies. ${ }^{15}$

In patients presenting with primary tumors, the inclusion of preoperative radiation in the management of these patients was associated with improved LC, with 5- and 10year LC rates of $85 \%$ and $71 \%$ with preoperative radiation, respectively, compared with $56 \%$ and $37 \%$ for exclusively postoperative radiation $(\mathrm{p}=0.019)$. Preoperative radiation doses of 50.4 GyRBE were generally used for mobile spinal tumors with few wound-healing complications. Because of the higher rate of wound-healing problems with sacral resections even in the absence of radiotherapy, ${ }^{13}$ the study authors have generally used only 19.8 GyRBE preoperative radiation for sacrococcygeal tumors, ${ }^{9}$ although they are currently evaluating 50.4 GyRBE preoperative radiation in conjunction with complex soft tissue or flap reconstructions for sacrococcygeal chordomas. ${ }^{4}$

The authors did not include patients in this study with

TABLE 6. Radiation-associated $\geq$ Grade 3 complications

\begin{tabular}{|c|c|c|}
\hline Complication & No. & Comment \\
\hline Wound infection after preop RT & 10 & Among 60 patients getting preop RT; $8 / 10$ had sacral tumors \\
\hline Wound dehiscence after preop RT & 3 & Sacral tumors \\
\hline Wound infection after op before RT & 7 & Among 58 patients getting only postop radiation \\
\hline Insufficiency fractures & 6 & 3 lumbar, 3 sacral; median RT dose 77.4 GyRBE \\
\hline Motor neuropathies & 4 & 3 lumbar; 77.4-85 GyRBE \\
\hline Spine nonunion \&/or hardware failure & 3 & Lumbar, 14-39 mos postop, median dose 70.2 GyRBE \\
\hline High-grade, radiation-associated soft tissue sarcoma & 1 & $\begin{array}{l}4 \text { yrs after preop RT, op, postop RT to } 70.2 \text { Gy to sacral chordoma; sarcoma treated } \\
\text { w/ chemotherapy, op }\end{array}$ \\
\hline Postop CSF leak after preop RT & 1 & Preop RT for locally recurrent chordoma after prior op \\
\hline Osteonecrosis & 1 & $\begin{array}{l}16.5 \text { yrs after postop RT } 66.6 \text { GyRBE; diabetic; flap necrosis after op for treatment of } \\
\text { osteonecrosis }\end{array}$ \\
\hline Rectal bleeding & 1 & $\begin{array}{l}\text { Preop RT, op, postop boost RT to } 70.2 \text { GyRBE; history of endometriosis, prior bowel } \\
\text { obstruction after earlier endometriosis op }\end{array}$ \\
\hline Late proctitis, rectal pain, tenesmus & 1 & $\begin{array}{l}13 \mathrm{~cm} \text { sacrococcygeal; preop RT, op, postop boost to } 70.2 \mathrm{GyRBE} \text {; rectum devascu- } \\
\text { larized at op; required rectosigmoid resection/colostomy }\end{array}$ \\
\hline Amenorrhea & 1 & Age 42; declined oophoropexy to move ovaries out of RT field \\
\hline Erectile dysfunction & 1 & $\begin{array}{l}\text { Sacral; erectile dysfunction } 3 \text { yrs after preop RT, op, postop boost RT to } 77.4 \text { GyRBE; } \\
\text { unresponsive to sildenafil }\end{array}$ \\
\hline
\end{tabular}


primary tumors who were undergoing nonsurgical management, because these patients were the focus of a prior study. ${ }^{7}$ Interestingly, LC at 5 years (with a median followup of 56 months) was $80 \%$ in the authors' previously published experience of 24 patients with primary chordomas managed by biopsy and high-dose proton-based radiation. ${ }^{7}$ This outcome was not markedly dissimilar to the excellent results in the current study noted with preoperative radiation, surgery, and postoperative RT boost, where LC at 5 years was $85 \%$ and was superior to that observed in patients undergoing surgery and postoperative radiation, in whom LC at 5 years was only 56\%. These are different retrospective data sets, and the comparison is offered to help clinicians and patients discuss and optimize treatment strategies and options for this challenging disease. Because preoperative radiation for primary tumor patients in conjunction with en bloc resection resulted in no local recurrences in the 28 patients so treated, that treatment appears to offer the highest rate of local tumor control, and it has been the authors' treatment recommendation for patients with lesions below S-3 where resection will not compromise anal or bladder sphincter control. For patients with upper sacral tumors with intact nerve function, the authors have discussed definitive radiation as an option, as well as offering this for patients who decline surgery. The authors' sense is that a nonsurgical approach would likely also be preferred for patients for whom R2 resections are considered or patients scheduled to undergo intralesional resections and postoperative radiation only. Definitive radiation without surgery, however, requires a higher radiation dose of approximately 77.4 GyRBE and carries a higher risk of nerve injury (estimated at $5 \%$ to $10 \%$ ), insufficiency fracture (33\%), and other potential treatment complications than the adjuvant dose of approximately 70.2 GyRBE used in conjunction with surgery. Hence, these treatment decisions often require making trade-offs between acute surgical morbidity and potential late radiation morbidity, as well as differential quality of life impacts. The authors have initiated a prospective quality of life study in this patient population to better inform these decisions (J. Schwab, personal communication).

While the treatments used in this study were successful in a significant proportion of patients, the median followup was 47 months, and it is important to acknowledge that although the rate of relapse decreases after 5 years, some patients will relapse after that time. These treatments can also be associated with both acute and late morbidity, as noted in the patients in this series who developed treatment-related complications, underscoring the need for ongoing improvements in surgery, radiation, and systemic therapies for this disease.

\section{Conclusions}

High-dose proton-based RT in the management of spine chordomas can be effective treatment. In patients undergoing surgery, patients with primary chordoma who undergo preoperative RT, en bloc resection, and postoperative RT boost have the highest rate of local tumor control. Intralesional and incomplete resections are associated with higher local failure rates and are to be avoided.

\section{Acknowledgments}

The authors wish to acknowledge Herman D. Suit, MD, $\mathrm{PhD}$, for his thoughtful discussions during the planning and preparation of this work. Financial support for this study was received in part by the Federal Share of program income earned by Massachusetts General Hospital on National Cancer Institute grant no. C06 CA059267, Proton Therapy Research and Treatment Center. Dr. Rotondo, Ms. Kobayashi, Dr. Chen, Ms. Szymonifka, Mr. Ferreira, and Dr. DeLaney received direct or indirect support from the Federal Share.

\section{References}

1. Arnautović KI, Al-Mefty O: Surgical seeding of chordomas. J Neurosurg 95:798-803, 2001

2. Baratti D, Gronchi A, Pennacchioli E, Lozza L, Colecchia $\mathrm{M}$, Fiore M, et al: Chordoma: natural history and results in 28 patients treated at a single institution. Ann Surg Oncol 10:291-296, 2003

3. Bergh P, Kindblom LG, Gunterberg B, Remotti F, Ryd W, Meis-Kindblom JM: Prognostic factors in chordoma of the sacrum and mobile spine: a study of 39 patients. Cancer 88:2122-2134, 2000

4. Bhatt AD, DeLaney TF, Schwab JT, Mullen JT, Liao EC, Ferreira A, et al: Reduction in wound complications with low- versus high-dose preoperative radiation therapy for sacro-coccygeal chordoma. Int J Radiat Oncol Biol Phys 87 Suppl:S166-S167, 2013 (Abstract \#1048)

5. Boriani S, Bandiera S, Biagini R, Bacchini P, Boriani L, Cappuccio M, et al: Chordoma of the mobile spine: fifty years of experience. Spine (Phila Pa 1976) 31:493-503, 2006

6. Boriani S, Saravanja D, Yamada Y, Varga PP, Biagini R, Fisher CG: Challenges of local recurrence and cure in low grade malignant tumors of the spine. Spine (Phila Pa 1976) 34 (22 Suppl):S48-S57, 2009

7. Chen YL, Liebsch N, Kobayashi W, Goldberg S, Kirsch D, Calkins G, et al: Definitive high-dose photon/proton radiotherapy for unresected mobile spine and sacral chordomas. Spine (Phila Pa 1976) 38:E930-E936, 2013

8. DeLaney TF, Chen GT, Mauceri TC, Munro JJ, Hornicek FJ, Pedlow FX, et al: Intraoperative dural irradiation by customized 192iridium and 90yttrium brachytherapy plaques. Int J Radiat Oncol Biol Phys 57:239-245, 2003

9. DeLaney TF, Liebsch NJ, Pedlow FX, Adams J, Dean S, Yeap BY, et al: Phase II study of high-dose photon/proton radiotherapy in the management of spine sarcomas. Int $\mathbf{J}$ Radiat Oncol Biol Phys 74:732-739, 2009

10. DeLaney TF, Liebsch NJ, Pedlow FX, Adams J, Weyman EA, Yeap BY, et al: Long-term results of Phase II study of high dose photon/proton radiotherapy in the management of spine chordomas, chondrosarcomas, and other sarcomas. J Surg Oncol 110:115-122, 2014

11. Fuchs B, Dickey ID, Yaszemski MJ, Inwards CY, Sim FH: Operative management of sacral chordoma. J Bone Joint Surg Am 87:2211-2216, 2005

12. Hanna SA, Aston WJ, Briggs TW, Cannon SR, Saifuddin A: Sacral chordoma: can local recurrence after sacrectomy be predicted? Clin Orthop Relat Res 466:2217-2223, 2008

13. Hulen CA, Temple HT, Fox WP, Sama AA, Green BA, Eismont FJ: Oncologic and functional outcome following sacrectomy for sacral chordoma. J Bone Joint Surg Am 88:1532-1539, 2006

14. Imai R, Kamada T, Tsuji H, Sugawara S, Serizawa I, Tsujii H, et al: Effect of carbon ion radiotherapy for sacral chordoma: results of Phase I-II and Phase II clinical trials. Int J Radiat Oncol Biol Phys 77:1470-1476, 2010

15. Kattapuram SV, Khurana JS, Rosenthal DI: Percutaneous needle biopsy of the spine. Spine (Phila Pa 1976) 17:561564,1992 
16. Ozaki T, Hillmann A, Winkelmann W: Surgical treatment of sacrococcygeal chordoma. J Surg Oncol 64:274-279, 1997

17. Park L, DeLaney TF, Liebsch NJ, Hornicek FJ, Goldberg S, Mankin H, et al: Sacral chordomas: impact of high-dose proton/photon-beam radiation therapy combined with or without surgery for primary versus recurrent tumor. Int J Radiat Oncol Biol Phys 65:1514-1521, 2006

18. Samson IR, Springfield DS, Suit HD, Mankin HJ: Operative treatment of sacrococcygeal chordoma. A review of twentyone cases. J Bone Joint Surg Am 75:1476-1484, 1993

19. Schwab JH, Healey JH, Rose P, Casas-Ganem J, Boland PJ: The surgical management of sacral chordomas. Spine (Phila Pa 1976) 34:2700-2704, 2009

20. Sciubba DM, Chi JH, Rhines LD, Gokaslan ZL: Chordoma of the spinal column. Neurosurg Clin N Am 19:5-15, 2008

21. Stacchiotti S, Casali PG: Systemic therapy options for unresectable and metastatic chordomas. Curr Oncol Rep 13:323-330, 2011

22. Yonemoto T, Tatezaki S, Takenouchi T, Ishii T, Satoh T, Moriya $\mathrm{H}$ : The surgical management of sacrococcygeal chordoma. Cancer 85:878-883, 1999

23. York JE, Kaczaraj A, Abi-Said D, Fuller GN, Skibber JM, Janjan NA, et al: Sacral chordoma: 40-year experience at a major cancer center. Neurosurgery 44:74-80, 1999

\section{Disclosure}

The authors report no conflict of interest concerning the materials or methods used in this study or the findings specified in this paper.

\section{Author Contributions}

Conception and design: DeLaney, Liebsch, Hornicek. Acquisition of data: DeLaney, Rotondo, Folkert, Ferreira, Hornicek. Analysis and interpretation of data: DeLaney, Rotondo, Liebsch, Chen, Schwab, Nielsen, Szymonifka, Hornicek. Drafting the article: Rotondo. Critically revising the article: DeLaney, Schwab, Hornicek. Reviewed submitted version of manuscript: DeLaney, Rotondo, Folkert, Liebsch, Chen, Pedlow, Schwab, Rosenberg, Nielsen, Ferreira, Hornicek. Approved the final version of the manuscript on behalf of all authors: DeLaney. Statistical analysis: Szymonifka. Administrative/technical/material support: Ferreira. Study supervision: DeLaney, Hornicek. Reviewed pathology specimens: Rosenberg, Nielsen.

\section{Supplemental Information}

Previous Presentation

Portions of this work were presented as abstracts at the following meetings: 2011 International Society of Limb Salvage Surgery Meeting, Beijing, China; 2011 Connective Tissue Oncology Society Meeting, Chicago, Illinois; 2011 American Society for Radiation Oncology Meeting, Miami, Florida; and 2014 Particle Therapy Cooperative Group Meeting, Shanghai, China.

\section{Correspondence}

Thomas F. DeLaney, Department of Radiation Oncology, Massachusetts General Hospital, 55 Fruit St., Boston, MA 02114. email: tdelaney@partners.org. 